\title{
Reliability assessment of existing bridge constructions based on results of non-destructive testing
}

\author{
Stefan Küttenbaum ${ }^{1, *}$, Alexander Taffe ${ }^{2}$, Thomas Braml $^{3}$, and Stefan Maack ${ }^{1}$ \\ ${ }^{1}$ Bundesanstalt für Materialforschung und -prüfung (BAM), Division 8.2, 12205 Berlin, Germany \\ ${ }^{2}$ HTW Berlin - University of Applied Sciences, 12459 Berlin, Germany \\ ${ }^{3}$ Bundeswehr University Munich, Institute of structural engineering, 85579 Neubiberg, Germany
}

\begin{abstract}
The non-destructive testing methods available for civil engineering (NDT-CE) enable the measurements of quantitative parameters, which realistically describe the characteristics of existing buildings. In the past, methods for quality evaluation and concepts for validation expanded into NDT-CE to improve the objectivity of measured data. Thereby, a metrological foundation was developed to collect statistically sound and structurally relevant information about the inner construction of structures without destructive interventions. More recently, the demand for recalculations of structural safety was identified. This paper summarizes a basic research study on structural analyses of bridges in combination with NDT. The aim is to use measurement data of nondestructive testing methods as stochastic quantities in static calculations. Therefore, a methodical interface between the guide to the expression of uncertainty in measurement and probabilistic approximation procedures (e.g. FORM) has been proven to be suitable. The motivation is to relate the scientific approach of the structural analysis with real information coming from existing structures and not with those found in the literature. A case study about the probabilistic bending proof of a reinforced concrete bridge with statistically verified data from ultrasonic measurements shows that the measuring results fulfil the requirements concerning precision, trueness, objectivity and reliability.
\end{abstract}

\section{Introduction}

Increasing requirements caused by heavy traffic coincide with both: the aging and the gradual degradation of bridge constructions in Germany. Recently, an increased demand for recalculations of structural safety was identified. The Eurocodes exclusively standardize the design of new constructions. Therefore, an adapted stepwise analysis for existing bridges was introduced [1]. The ultimate level of this analysis permits the application of scientific procedures, e. g. probabilistic methods.

The main difference in comparison to the semiprobabilistic approach is that random variables are input quantities. Safety elements such as partial safety indexes or characteristic values are not included. This means that individual uncertainties coming from the structural models, material properties or geometrical dimensions must be implicitly included in the stochastic variables. Uncertainties are not covered by empirically derived and standardized safety elements.

It is obvious that the accuracy of results cannot be better than the accuracy of the input values, but the data base for the stochastic modelling in civil engineering is insufficient. One reason may be the uniqueness of buildings and the question about the assignability of data from one construction to another.
Assuming that the decision-making process, whether a construction is stable or not, depends on the structural engineer's knowledge about the existing building, real structural properties should be used for the evaluation (Fig. 1). For example, the quantification of influences on the stochastic model based on the results of visual bridge inspections is shown in [2] to expand this knowledge.

This paper summarizes the approach, to use quantitative data in structural analyses which were gained from non-destructive measurements. On the one hand, probabilistic methods allow static calculations involving measurement data. On the other hand, the application of measured data reduces uncertainties in structural analyses. This requires statistically sound data. The realisation and advantages of non-destructive measured data in static calculation are described below.

\section{Non-destructive testing of bridges}

Non-destructive testing (NDT) may be defined as a "technical process to quantify characteristic values of a material (...) according to a certain procedure using the interaction of energy and material-property without affecting the serviceability." [3] In NDT in civil engineering (NDT-CE), it may be distinguished between classical (e.g. rebound hammer) and advanced methods.

* Corresponding author: stefan.kuettenbaum@bam.de 
Quantitative as well as qualitative information about the inner structure are gained by the application of those techniques. In the past, this knowledge was mainly used to analyse damages. In the following, ultrasound is used to illustrate a NDT-based evaluation of structural safety.

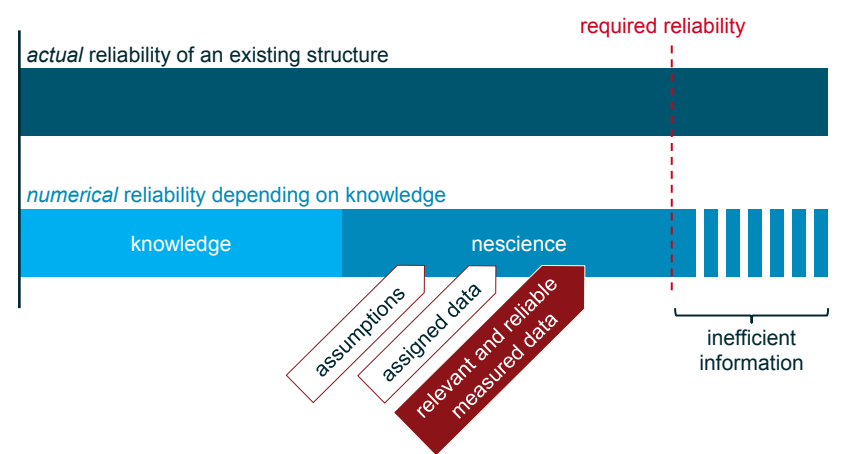

Fig. 1. Ways to collect necessary information about existing structures to reach sufficient reliability

\subsection{Typical testing problems for ultrasonic echo}

Ultrasonic pulse echo has been proven to be suited for different testing tasks on reinforced and pre-stressed concrete bridges. Fig. 2 shows typical testing tasks.

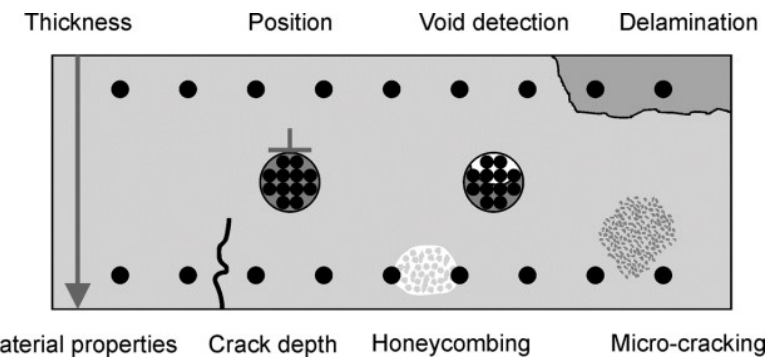

Fig. 2. Typical testing tasks for ultrasonic echo on reinforced concrete including tendon ducts [4]

Ultrasonic echo is principally based on the measurement of the transit time. The aim is to determine the depth $d$ and the lateral position of a reflector. The target value $d$ is a variable depending on the measured transit time $t$ of the transmitted, reflected and received pulse (back on the surface) as well as the sound velocity $v$, which depends on material properties.

$$
d=v_{\text {material }} \cdot t / 2
$$

Basic information about the physical principles of ultrasonic measurements and NDT-CE in general are given in [4].

\section{Application on bridge constructions}

Target values of ultrasonic echo measurements may be heights and widths of bridge superstructures or hollow boxes, the position and the grouting condition of tendon ducts as well as the concrete cover depths. Due to the acoustic impedances of steel and concrete, ultrasonic waves may also give information about reflectors behind steel. Therefore, the centre of reinforcement can be determined if more than one layer of reinforcement is present. Thereby, the effective depth of a cross-section can be gained by ultrasonic measurement data.

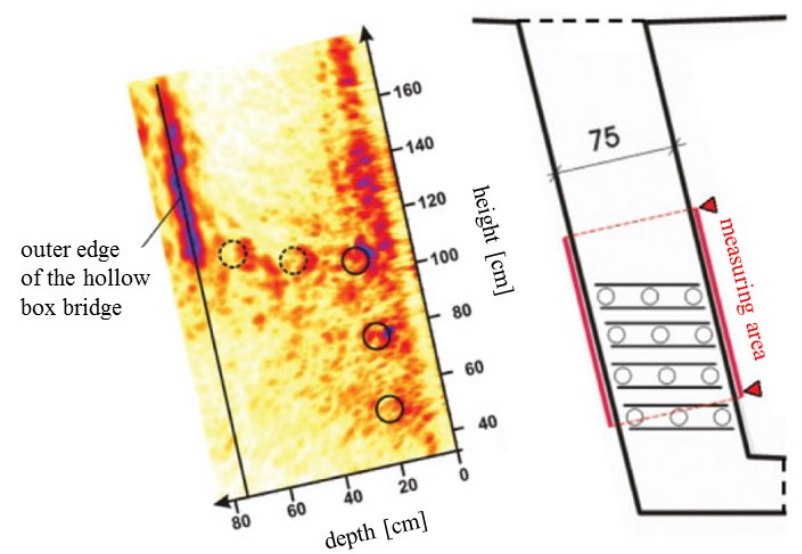

Fig. 3. SAFT-reconstructed ultrasonic measurement data and the cross section of a hollow box girder web [5], translated

An example for an ultrasonic measurement result at a hollow box girder web is shown in Fig. 3. The measured data were reconstructed by using the SAFT-algorithm [6]. Even if other reflectors were in front, the tendon ducts could be detected up to a depth of approximately $55 \mathrm{~cm}[5]$.

\subsection{Statistically sound measuring data}

The circles shown in Fig. 3 symbolise the detected tendon ducts with deterministic depths, whereas the measurement must be considered as random experiment: In general, the measurement data are scattered by random errors. Therefore, the illustration of those tendon ducts in combination with their individual measurement accuracy would be more helpful to improve the objectivity of measurements and to specify structurally conservative results. The aim is to evaluate the accuracy of a measurement.

In the following, the standard deviation quantifies the scattering and expresses the precision of a measurement. In contrast, systematic errors affect the trueness of a measurement. Wrong data can be improved by correct calibration. They may be illustrated as a probability density function (PDF) with a shifted mean value. The accuracy can be defined as a collective term for both trueness and precision [7] (Fig. 4).

Results of measurements should be adjusted for systematic deviations. This means, that the measurement accuracy has to be evaluated by the expression of this corrected true result in combination with the uncertainty in measurement. It is important to bear in mind, that precise but wrong measurements harbour the risk, that deviations remain undetected. 


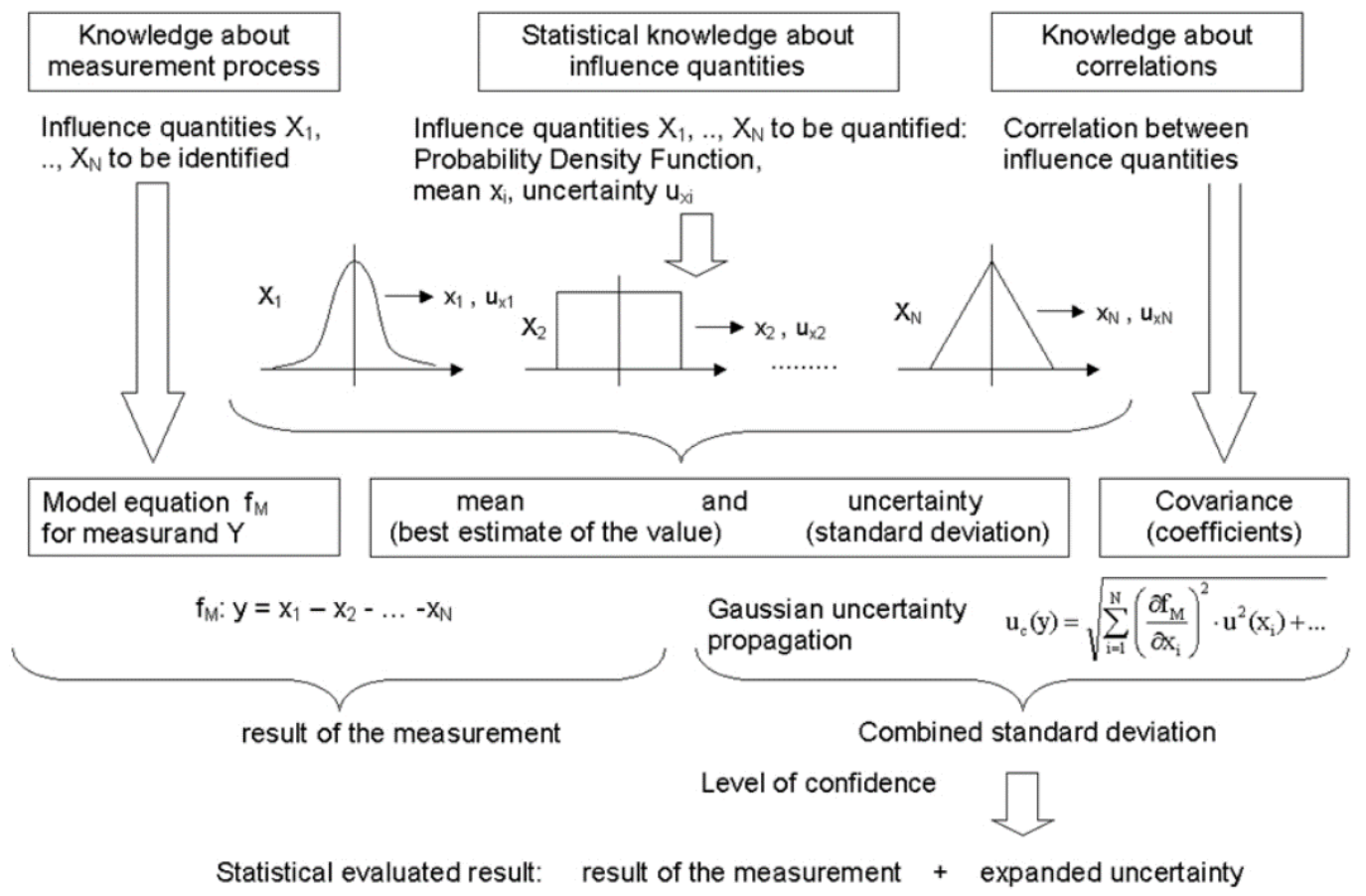

Fig. 5. Steps to be taken for method characterisation by following the GUM; extracted from [11], according to [10] and [12]
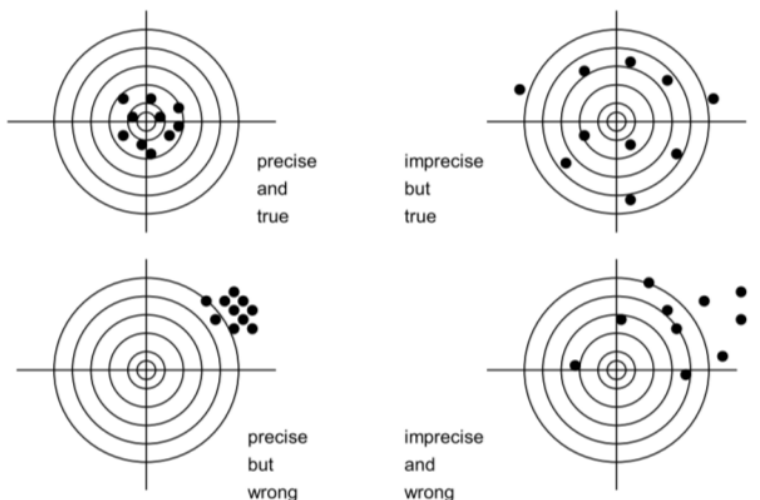

Fig. 4. Trueness and precision illustrated in a target model; a true value is expressed in the centre of the targets [7]

Those considerations are taken over to NDT-CE to express uncertainties in quantitative as well as in qualitative measurements [8]. The Guide to the Expression of Uncertainty in Measurement (GUM) [9] has been proven to be well suited for the evaluation of quantitative measurement data [10], e.g. the position of tendon ducts. The basic concept is to identify the influence quantities $X_{i}$ on the displayed measured value. An Ishikawa diagram can be used to plot those influences. Knowledge about the measurement process is necessary for that. This is followed by the definition of a well fitted PDF and the quantification of statistical moments for all those single deviations. Furthermore, an equation should bring the influence quantities into a functional context. This results in a model equation, e.g. of thickness measurements for ultrasonic echo (eq. 1) using transverse waves: $d(t, v)=(v+\delta v) \cdot\left(t_{\text {measured }}-\delta t_{\text {device }}-\delta t_{\text {material }}-\delta t_{\text {specimen }}\right) / 2$

In eq. 2 the transit time $t_{\text {measured }}$ is the displayed value, the variables $\delta t_{i}$ are deviations coming from e. g. the limited resolution of the time axis, the variation of the sound speed in the inhomogeneous concrete and unevenness surfaces [10].

The quantity value of the measurand $d$ can be obtained from eq. 2 and represents the measurement result. Applying the uncertainty propagation to this model function leads to the combined standard deviation $u(y)$. In addition, a coverage interval must be expressed considering the explicit treatment of the accuracy evaluation. Therefore, the combined standard deviation $u(y)$ is multiplied with an enhancement factor $k$. The GUM recommends a coverage probability of $95 \%$. Assuming a normally distributed measurand, this level of confidence is reached by a multiplication with $k \approx 2$. This leads to the expanded uncertainty $U(y)$.

The corrected measurement result has to be assigned to both the expanded uncertainty as well as to the coverage probability in order to express the statistically evaluated result clearly.

The procedure is comprehensive and single deviations can be adapted to other test conditions. The whole method characterisation process is illustrated as a flowchart in Fig. 5.

\section{Probabilistic structure analyses}

This chapter describes how statistical sound (stochastic) measuring data can be included into structural analyses. The approach with probabilistic methods and stochastic input variables is well suited, for instance to enter 
multivariate target values into the static calculation. Those target values result from more than one NDTmeasurand (e. g. the permanent load derived from geometrical dimensions). They cannot be calculated backwards positively to semi-probabilistic values.

\subsection{Concept of structural safety}

An easy approach to illustrate the idea of probabilistic methods in structural analyses is the comparison of the effects of actions $E$ and the resistance $R$. In this case both should be understood as random variables with Gaussian distribution. The functional relationship between $E$ and $R$ is called limit state function and is generally given by:

$$
g(E, R)=\mathrm{R}-\mathrm{E}
$$

As drawn in Fig. 6, the areas under the curves of $E$ and $R$ generate an interception. This area is synonymous to the possibility, that the effects of actions reach higher values than the resistance of the structure. This would lead to a structural failure. Thus, the probability of failure $p_{f}$ can be determined by solving:

$$
p_{f}=\mathrm{p}(\mathrm{g}<0)
$$

This probability is the fundamental quantity to prove the structural safety based on the reliability theory.

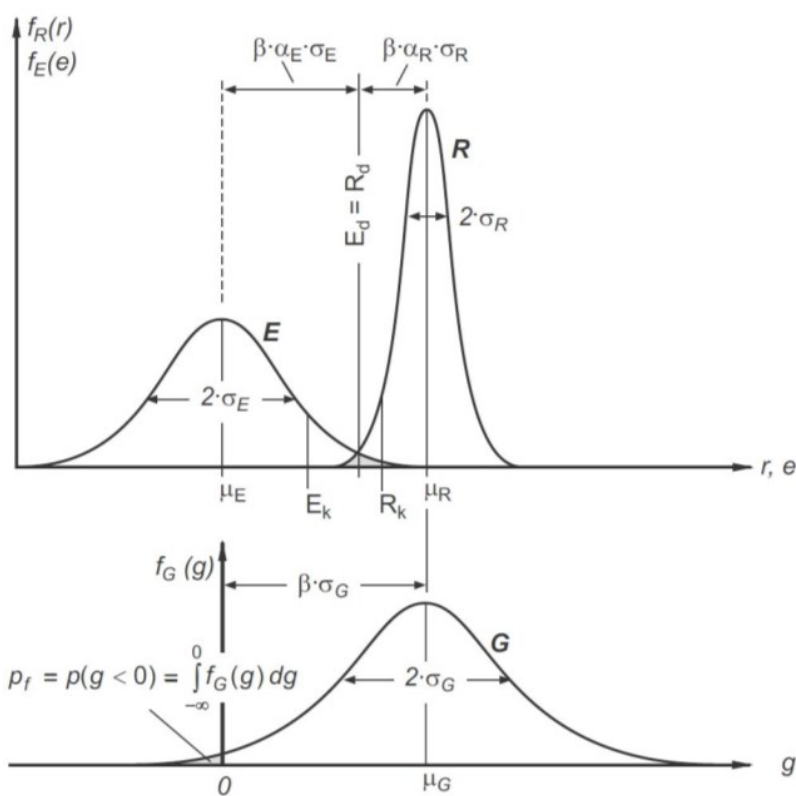

Fig. 6. Safety concept illustrated by comparing the effects of actions $E$ and the resistance $R$ and associated with the (wellknown) characteristic values $X_{k}$ and design values $X_{d}$ [13]

The reliability of a construction is the complement to the probability of failure. The generalised reliability index $\beta$ is equivalent to $p_{f}$ and should ensure a minimum distance between the mean values $\mu_{E}$ and $\mu_{R}$. The calculated index $\beta$ depends on the standard deviations $\sigma_{i}$ and the sensitivity factors $\alpha_{i}$ of the random variables.
This short overview should outline the importance of accurate input variables in an explicit proof of the probabilities of failure. Wrong assumptions influence the results of the structural analysis - consciously or unconsciously. Safeguarding by standardized safety elements are no longer given in comparison to the semiprobabilistic design of new buildings.

Further information about the safety concept and the reliability theory in structural engineering can be taken from e.g. [14].

\subsection{Diagnostic tools}

A key advantage of applying probabilistic principles for analyses of structural safety is the possibility to use sensitivity analyses and parametric studies for the interpretation of input values and their influence on determined reliabilities.

The shortly introduced sensitivity factors are results from sensitivity analyses. Those $\alpha_{i}$ are depending on the standard deviations of the stochastic input variables. The idea is to determine how sensitive the individual reliability reacts to the variation of the statistical scattering of a random variable. A small value reveals that the random variable may be modelled with less precision without significant loss of reliability.

Therewith conclusions may be drawn e.g. to plan a systematic update of the primary stochastic model considering building inspections. The sum of squares of all sensitivity factors is one. Therefore, the results can be illustrated in a pie chart. The algebraic sign of a sensitivity factor provides the information whether an input variable appears stabilising (positive values) or destabilising (negative values).

Another result of probabilistic analyses are so-called elasticities. Their informative value about the input values is similar to the sensitivity factors. A difference is that the elasticities might be related to mean values and to standard deviations as well. They express changes in reliability as a consequence of varying one of both statistical moments by one percent. An advantage is that the elasticities enable quantitative interpretations without advanced considerations. Further, they do not react as susceptible as the sensitivity factors to correlations.

Finally, the following case study accesses parametric studies as a third diagnostic tool. The purpose is to graph the curve of the reliability index as a function depending on the variation coefficient of one stochastic variable. This way an individually necessary precision (to reach the required target reliability) may be deviated for each random variable. In the context of measurands as input variables in structural analyses, the parametric studies can be used for the specification of the sufficient quality of measurements.

\section{Example: Reinforced concrete bridge}




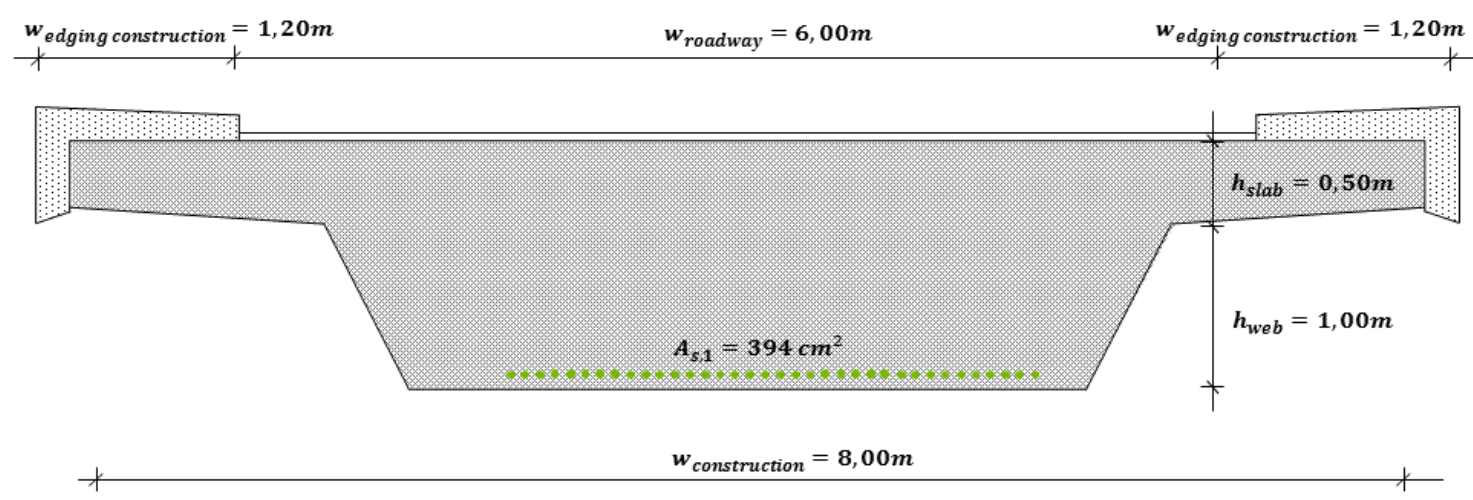

Fig. 7. Dimensional sketch of the considered massive plate cross section [15], translated

This case-study deals with a single beam. It has a span of twenty meters and a cross section formed as massive plate. The concrete bridge is equipped with reinforcement bars subjected to tensile load. The decisive cross section is drawn below in Fig. 7. The aim is to prove bending to illustrate the practicability and the advantages of structural analyses considering measured data gained from ultrasonic pulse echo technique.

Solving the equilibrium criteria considering the cracked stated cross section results in the following design value of the resisting internal bending moment:

$$
M_{R d}=A_{s 1} \cdot \frac{f_{y k}}{\gamma_{s}} \cdot d \cdot\left(1-\frac{k_{a}}{\alpha_{R}} \cdot \frac{A_{s 1} \cdot f_{y k} \cdot \gamma_{c}}{b \cdot d \cdot \alpha \cdot f_{c k} \cdot \gamma_{s}}\right)
$$

It was mentioned before, that partial safety indexes $\gamma_{i}$ must be substituted. Instead of that, uncertainties of the structural models and loads $\Theta_{i}$ were added. Assuming the efficient equation of $E=R$, so that the reinforcement is fully loaded, and using the simplified constant stressstrain-relation to eliminate surplus unknowns, the function $G=R-E$ of the ultimate limit state (ULS) bending results in:

$$
\begin{aligned}
G=\Theta_{R, M} \cdot A_{s 1} \cdot f_{y} \cdot d \cdot\left(1-\frac{A_{s 1} \cdot f_{y}}{2 \cdot \chi \cdot b \cdot d \cdot \alpha \cdot f_{c}}\right) \\
-\Theta_{E, M} \cdot\left(M_{G}+M_{Q}\right)
\end{aligned}
$$

The formula symbols are corresponding with the European design standards [16]. To be able to follow this text, it is enough simply to know that

$d \quad-$ effective depth of a cross-section,

$M_{G} ; M_{Q}$ - dead loads; traffic loads,

$\Theta_{R} ; \Theta_{E}$ - uncertainty of resistance; of load effects.

Next, the input values in eq. 6 must be stochastically modelled if possible. Those random variables are also called basis variables. Overall, they form the assessment basis, which reveals the needed information about the construction to prove the ULS bending. At this point the structural engineer has to decide whether he uses values found in the literature or data gained from previous, individual bridge inspections (combined as necessary) to get this information.

The presented approach pursues the objective to enable the engineer to perform the second type of stochastic modelling (with relevant and reliable measured data) For this purpose and to avoid destructive interventions, knowledge about the measurement principles and processes allows the identification of nondestructive measurable basis variables, in this case determined by ultrasonic testing. In addition, the provided structurally relevant input values in eq. 6 must be aligned with the question about how those basis variables may be reasonably measured at the individual structure in order to reach sufficient precision. This way, a suitable measurement strategy can be obtained. The procedure reveals if basis variables are direct measurands or indirect target values. The outcome of this is an adapted assessment basis for this individual analysis and a modified limit state function considering the non-destructive issues. Thereby the statistically sound measured data are calculable.

A simplified example should point out the procedure: The bending proof requires information about the dead loads $M_{G}$. Those are depending on densities and volumes of concrete and reinforcement. Whereas the ultrasonic echo technique is not suitable for density measurements, the measured geometrical dimensions may be used to evaluate stochastic models for the volumes of steel and concrete. The volume of concrete itself is a multivariate target value consisting of different thickness measurements. Expanding the basis variable dead loads into measurable thicknesses is equivalent to expand the assessment basis.

Another example should illustrate the advantages of knowledge about the inner construction gained by NDT considering the cross section in Fig. 7. Subtracting the height of the construction and the distance between the lower edge and the centre of the reinforcement results in the effective depth of the cross-section $d$. The ultrasonic echo technique may give the required information about the position of the bars. The following structural analysis shows the significance of $d$.

The derivation of quantitative uncertainties in measurement must be excluded from this contribution as well as the correlation matrix although the quality of input parameters significantly affects the informative value of probabilistic analyses. The following extract of the stochastic model tabulates the measurement precision of $d$ exemplarily as a result obtained by individually evaluated uncertainties in ultrasonic testing. 
Table 1. Extraction of the stochastic model, the NDTmeasurand $d$ was modelled with uncertainties based on GUM, $\mathrm{LN}=$ Log-normal distribution, $\mathrm{N}=$ Gaussian distribution

\begin{tabular}{|c|c|c|c|c|}
\hline & $\begin{array}{c}\text { distri- } \\
\text { bution }\end{array}$ & $\begin{array}{c}\text { mean } \\
\text { value }\end{array}$ & $\begin{array}{c}\text { coeff. } \\
\text { of var. }\end{array}$ & $\begin{array}{c}\text { refe- } \\
\text { rence }\end{array}$ \\
\hline $\begin{array}{c}\text { uncertainty of } \\
\text { resistance }\end{array}$ & $\mathrm{LN}$ & 1,20 & $15,0 \%$ & {$[19]$} \\
\hline $\begin{array}{c}\text { area of } \\
\text { reinforcement } A_{s l}\end{array}$ & $\mathrm{~N}$ & $\begin{array}{c}0,0394 \\
\mathrm{~m}^{2}\end{array}$ & $2,0 \%$ & {$[20]$} \\
\hline $\begin{array}{c}\text { effective depth of } \\
\text { the cross-section } d\end{array}$ & $\mathrm{~N}$ & $\begin{array}{c}1,40 \\
\mathrm{~m}\end{array}$ & $6,4 \%$ & $\begin{array}{c}\mathrm{GUM}- \\
\text { based } \\
{[10]}\end{array}$ \\
\hline $\begin{array}{c}\text { compression } \\
\text { concrete strength } f_{c}\end{array}$ & $\mathrm{~N}$ & $\begin{array}{c}40.000 \\
\mathrm{kN} / \mathrm{m}^{2}\end{array}$ & $7,5 \%$ & {$[2]$} \\
\hline
\end{tabular}

\section{Results of the probabilistic analysis}

The analyses were executed by software [18]. The structural safety has been proven. The structural analysis of ULS bending in midspan results in a calculated reliability index $\beta \approx 5,0$, greater than the required value of $\beta=3,8$. Furthermore, scientific conclusions may be derived from the diagnostic tools, beginning with the sensitivity analysis:

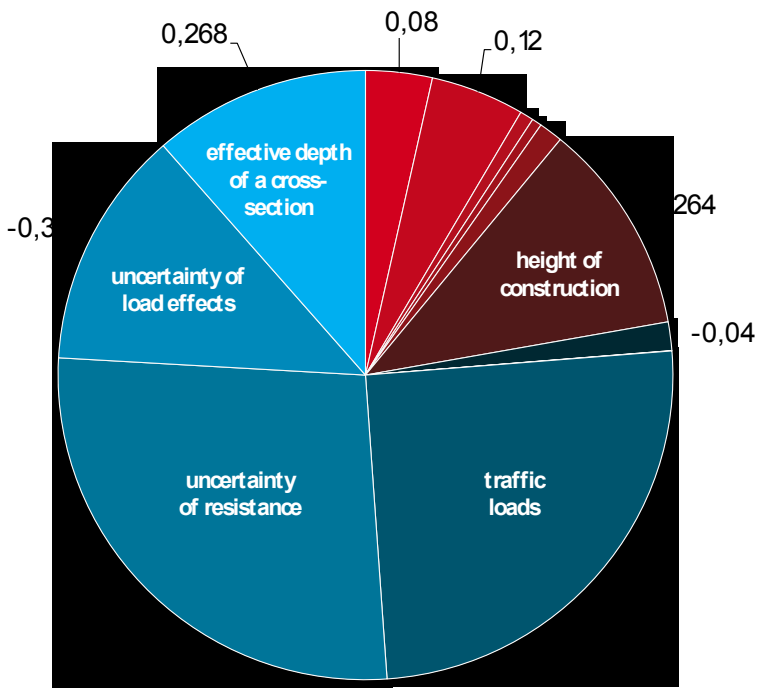

Fig. 8. Sensitivity factors [17]
As already mentioned before the sensitivity factors provide the decisive input variables in probabilistic analyses of structural safety. The pie chart in Fig. 8 highlights the great influence on the limit state based on model uncertainties of resistance as well as of load effects. In contrast, the calculated reliability reacts less sensitive to those random variables which were stochastically modelled on the basis of statistically sound measured NDT-data. Both the effective depth and the height of construction become noticeable. The last one just attaches a significant value due to correlation effects. In most cases the precision of ultrasonic pulse echo may be classified as accurate enough.

The elasticities give further diagnostic information and can be used to draw two different key conclusions. The values are shown in Fig. 9. The elasticities of mean values are between $-0,79<\mathrm{e}_{\mu, \mathrm{i}}<1,32$ in this case. Those results express the appropriate input parameters that should be remodelled regarding the mean to provide a proof efficiently while designing a new building or planning structural reinforcements. Metrologically this first way of interpretation is not as meaningful. Changes in the mean value may especially occur while correcting systematic deviations. Those calibration procedures must be finished before entering the statistical moments into the reliability analysis, so that trueness is obtained.

In this context the elasticities of standard deviations $\mathrm{e}_{\sigma, i}$ have a greater informative value. The higher the absolute value, the bigger the influence of a random variables scattering on the reliability. In the present case those elasticities reach values up to $e_{\sigma, i} \geq-0,45$. Usually they are negative because increasing standard deviations should logically cause decreasing reliability. The results for the elasticities of standard deviation are consistent to the illustrated sensitivity factors in Fig. 8. The single exception is the height of construction $\left(\alpha_{h} \approx 0,26\right.$; $\mathrm{e}_{\sigma, \mathrm{h}} \approx 0$ ) due to numerical issues.

Regarding the application of measured data in this analysis, the elasticities of standard deviations express the consequences of increasing random errors (decreasing precision) in measurements on the calculated reliability. From the metrological point of view, this is the proper key conclusion gained from the elasticities, because the precision of measured data is adjustable by varying boundary conditions (e.g. the measuring grid). 


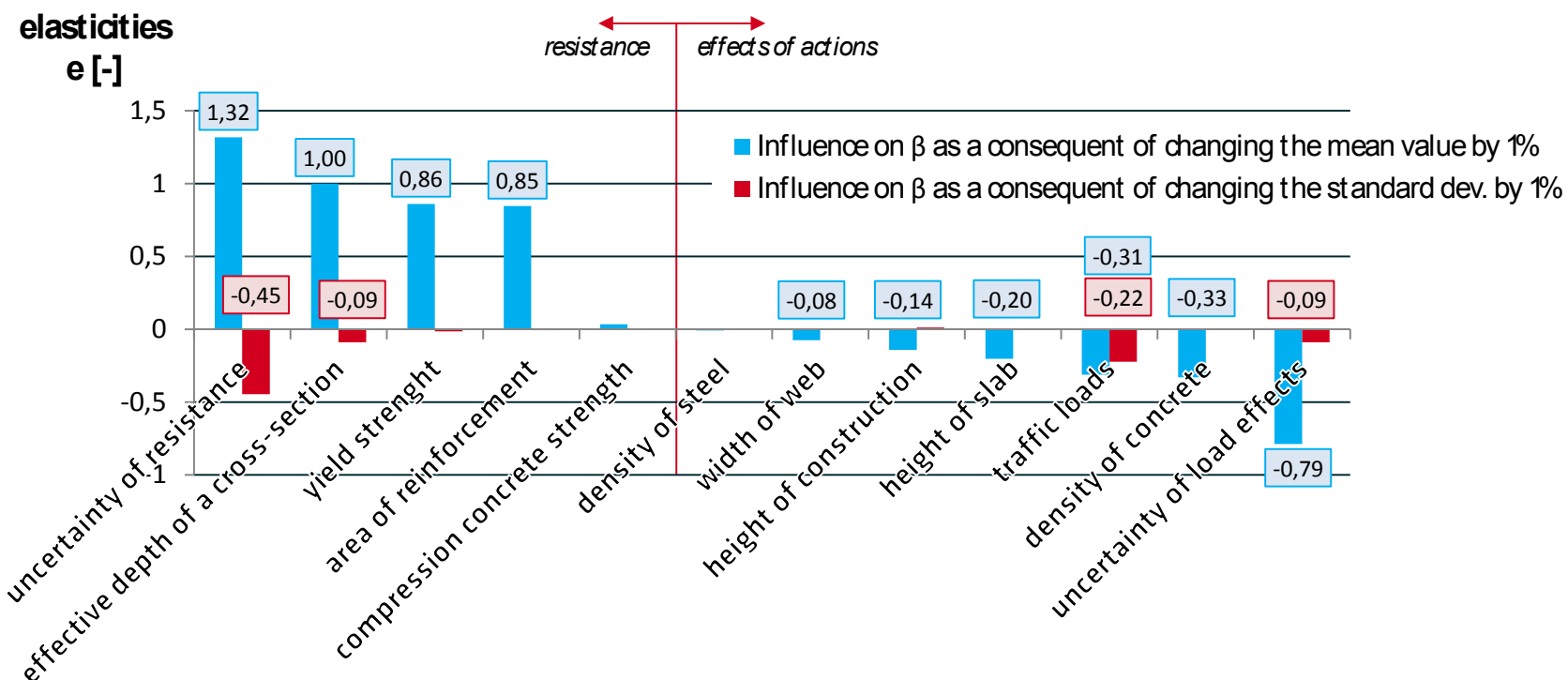

Fig. 9. Elasticities gained by varying statistical moments of each basis variable [17]

The single basis variable out of all NDT-data based random variables with a significant influence on the ULS bending is the effective depth of the cross-section $d$ according to $\alpha_{d} \approx 0,27$ and $e_{\sigma, d} \approx-0,09$. Thus, particularly the systematic variation of the scattering characteristic of $d$ and the examination of existing reliabilities seem to be likely. The results of those parametric studies may be illustrated as a course of the reliability function depending on the value of the variation coefficient $v_{d}$. This relative scattering dimension is varied between $1 \% \leq \mathrm{v}_{d} \leq 30 \%$. The curve is shown in Fig. 10. The used expanded uncertainty (relating to the mean) is $v_{d}=6,4 \%$. The triangle points out this evaluated precision. The value just applies to the individually considered boundary conditions in this structural analysis.

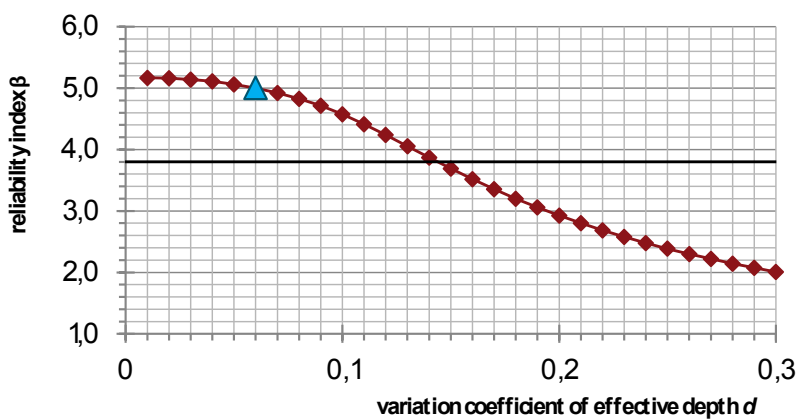

$\longrightarrow$ calculated reliability

required reliability $(\mathrm{RC} 2)$

Fig. 10. Reliability trend while changing the variation coefficient of the effective depth of the cross-section [17]

The course of the function proves not only that the determined uncertainty in ultrasonic testing leads to sufficient reliability but also that a less precise measurement would not implicitly cause a numerical failure. This information should be used to answer the already mentioned question about the needed precision. This way, the primary stochastic model may be updated efficiently by specifically defined bridge inspections and measurement strategies.
This way we derive requirements towards the measurement accuracy from structural analyses. Furthermore, the curve motivates to link the scattering characteristics of $d$ or other random variables with NDTmethods, measurement strategies, influencing boundary conditions etc. All of them have to be suitable to reach the particular precision.

The parametric studies relate different curves to each single basis variable. For instance, no noticeable impact on the reliability can be identified while changing the variation coefficient of the height of slab. This height was also stochastically modelled with NDT-data (Fig. 11). In comparison to the results drawn before, the measurement of the height of slab with significantly less precision would not affect the numerical reliability.

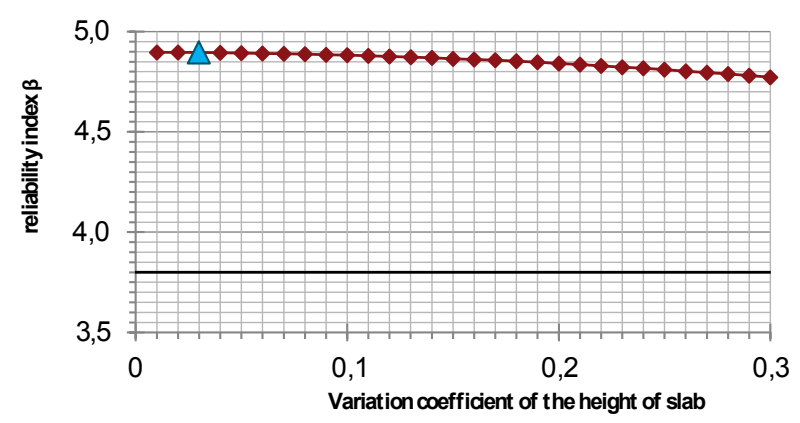

Fig. 11. Reliability trend while changing the variation coefficient of the height of slab [17]

\section{Conclusion}

It has been shown that (scattering) statistically sound measured data which were gained from non-destructive testing may be used as input values in probabilistic structure analyses. The application of quantitative NDTmeasurements improves the knowledge of the structural engineer. Furthermore, the ultrasonic pulse echo results are proved to be well suited for the application in static calculations. They fulfil the requirements concerning the precision, trueness, objectivity and reliability. It has been 
analysed that the structural analysis results in sufficient reliability without measuring as accurately as possible.

The further development of this research project particularly contains interconnected subprojects: Firstly, other ultimate and serviceability limit states will be examined as well as further NDT-methods. Secondly, a practical example on a reinforced concrete bridge should show the practicability and the advantages of the presented approach.

Structural engineers usually aspire toward greater calculative reliabilities for existing structures until the required target value could be proven. This effort relates to the introduced ways of gathering information to reach sufficient reliability in the beginning. Fig. 12 illustrates in this context, how increasing knowledge, expressed as input variables with a higher precision, affects the reliability index positively.

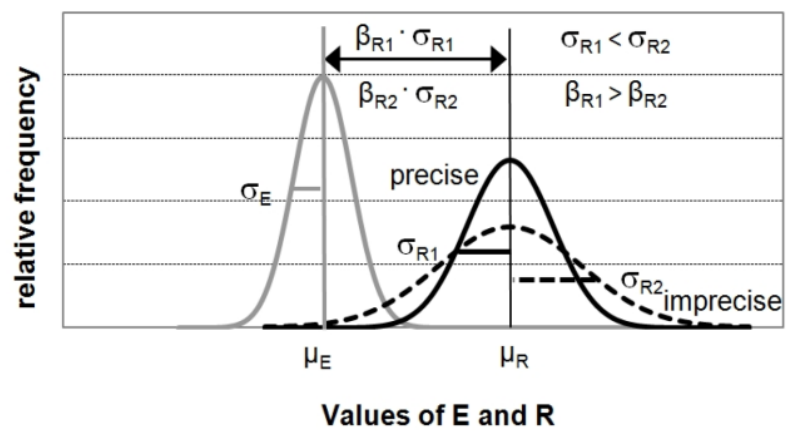

Fig. 12. Effect of precise knowledge on the reliability [11]

The informative value of probabilistic methods directly depends on the quality of measurements and of collected data. It was not researched exhaustively until now, that NDT-methods can give more accurate information about structure properties than the already known and more or less universally valid values found in the literature. Therefore, the closing issue may have a philosophical trait: What kind of input quantities creates safer knowledge than realistic and statistically sound characteristics of the individual existing construction ideally collected without destructive interventions.

\section{References}

[1] Federal Ministry of Transport, Building and Urban Development: Richtlinie zur Nachrechnung von Straßenbrücken im Bestand (2011) and 1st supplement (2015)

[2] Th. Braml: Zur Beurteilung der Zuverlässigkeit von Massivbrücken auf der Grundlage der Ergebnisse von Überprüfungen am Bauwerk, Fortschrittberichte VDI 214 (Reihe 4), VDI Verl., Düsseldorf (2010)

[3] H.-U. Richter: Chronik der zerstörungsfreien Materialprüfung, DVS, Berlin (1999)

[4] M. Schickert, M. Krause: Ultrasonic techniques for evaluation of reinforced concrete structures. In: Ch. Maierhofer, H.-W. Reinhardt, G. Dobmann (eds.): Non-destructive evaluation of reinforced concrete structures. Woodhead Publishing Ltd., Cambride (2010), Part II.22, pp. 490-530

[5] D. Streicher, C. Kohl, H. Wiggenhauser, A. Taffe: Automatisierte zerstörungsfreie Zustandsuntersuchungen von Brückenbauwerken. In: Beton- und Stahlbetonbau 101 (2006) 5, pp. 330-342

[6] M. Schickert, M. Krause, W. Müller: Ultrasonic Imaging of Concrete Elements Using Reconstruction by Synthetic Aperture Focusing Technique. In: J. of Non Destructive Evaluation 27 (2008), pp. 35-45

[7] W. Hässelbarth: Guide to the Evaluation of Measurement Uncertainty for Quantitative Test Results, EUROLAB Technical Report, Paris (2006)

[8] A. Taffe, S. Feistkorn: Methoden zur Gütebewertung von ZfPBau-Verfahren. In: Betonund Stahlbetonbau 108 (2013) 4, pp. 237-251

[9] JCGM 100:2008 Evaluation of measurement dataGuide to the expression of uncertainty in measurement

[10]A. Taffe: Zur Validierung quantitativer zerstörungsfreier Prüfverfahren im Stahlbetonbau am Beispiel der Laufzeitmessung, DAfStb-Heft 574, Beuth, Berlin (2008)

[11] T. Braml, A. Taffe, S. Feistkorn, O. Wurzer: Assessment of Existing Structures using Probabilistic Analysis Methods in Combination with Nondestructive Testing Methods. In: Structural Engineering International 23 (2013) 4, pp. 376-85.

[12] K.-D. Sommer, B. Siebert: Systematic approach to the modelling of measurements for uncertainty evaluation. In: Metrologia 43 (2006) 4, pp. 200-210.

[13]K. Zilch, G. Zehetmaier: Bemessung im konstruktiven Ingenieurbau, 2nd edition, Springer, Berlin, Heidelberg (2010)

[14]G. Spaethe: Die Sicherheit tragender Baukonstruktionen, 2nd edition, Springer, Wien, New York (1992)

[15]A. Taffe, S. Küttenbaum, S. Maack: Verwendung von Messergebnissen zerstörungsfreier Prüfverfahren im Bauwesen in statischen Nachweisen. Statistisch abgesicherte Messgrößen beschreiben dem Prüfingenieur die tatsächlich ausgeführte Konstruktion. In: Der Prüfingenieur 52 (2018), ISSN 1430-9084 (in print)

[16]DIN EN 1992-2:2010-12: Eurocode 2: Design of concrete structures - Part 2: Concrete bridges Design and detailing rules; German version EN 1992-2:2005 + AC:2008

[17]S. Küttenbaum: Beitrag zur Verwendung von Messergebnissen zerstörungsfreier Prüfverfahren in statischen Berechnungen - Aufstellen von Grenzzustandsgleichungen, Master-Thesis, Berlin (2017)

[18] Reliability Consulting Programs (RCP GmbH): STRUREL, structural reliability analysis programsystem, COMREL v. 9.0, Munich

[19]A. M. Fischer: Bestimmung modifizierter Teilsicherheitsbeiwerte zur semiprobabilistischen Bemessung von Stahlbetonkonstruktionen im Bestand, Dissertation. Kaiserslautern (2010) 
[20] Joint Committee on Structural Safety: JCSS Probabilistic Model Code, Part 3: Material Properties (2002) 